Supporting Information

\title{
Assembly of Interlocked Superstructures with a Titanium-Oxide Molecular Ring in Water
}

\author{
Caiyun Liu, ${ }^{\mathrm{a}}$ Chang Gao, ${ }^{\mathrm{a}}$ Amir Said, ${ }^{\mathrm{a}}$ Huihui Niu, ${ }^{\mathrm{a}}$ Dexin Wang, ${ }^{\mathrm{a}}$ Chen-Ho Tung ${ }^{\mathrm{a}}$ and Yifeng Wangab* \\ ${ }^{a}$ Key Lab for Colloid and Interface Science of Ministry of Education, School of Chemistry and Chemical \\ Engineering, Shandong University, Jinan 250100, China \\ ${ }^{b}$ State Key Laboratory of Crystal Materials, Shandong University, Shanda South Road 27, $250100 \mathrm{Ji}$ - \\ nan, China
}

\section{Table of contents}

\section{Experimental section}

\section{Supplementary Figures}

Figure S1. The photos of the compounds.

Figure S2. Raman spectra of the compounds.

Figure S3. Diffuse reflectance UV-vis spectra of the compounds.

Figure S4. IR spectra of [Tis $\cdot$ EtDA], $\left[\mathbf{T i}_{8} \cdot \mathbf{C}_{8} \mathbf{N}_{6} \mathbf{P y}_{2}\right]$ and $\left[\mathbf{T i}_{8} \cdot \mathbf{C}_{30} \mathbf{N}_{8}\right]$.

Figure S5. SAXS data for [Ti8.EtDA] in water.

Figure S6. ${ }^{1} \mathrm{H}$ DOSY NMR of EtDA and [Tis·EtDA].

\section{References}




\section{Experimental section}

Materials. $\mathrm{TiCl}_{4}, \quad \mathrm{TiOSO}_{4}, \quad \mathrm{Li}_{2} \mathrm{SO}_{4}, \mathrm{H}_{2} \mathrm{SO}_{4}$ (aq), polyethylene polyamine (PEPA), bis(3aminopropyl)amine, 1,4-phthaladehyde, benzaldehyde and pyridine-4-carboxaldehyde were obtained from Aladdin Industrial Inc and used as received. The linear amines, like ethylenediamine (EtDA), propanediamine (PrDA), butanediamine (BuDA), triethylenetriamine (TEtTA) and spermidine were of analytical grade, obtained from commercial sources and used as received. The commercially unavailable amines were prepared with the procedure reported later. All solutions were prepared using $18.25 \mathrm{M} \Omega$ deionized water.

Instruments. Single crystal X-ray diffraction data were collected using a Rigaku Oxford Diffraction XtaLAB Synergy diffractometer equipped with a HyPix-6000HE Hybrid Photon Counting (HPC) detector operating in shutterless mode and an Oxford Cryosystems $800 \mathrm{Plus} \mathrm{Cu} K \alpha(\lambda=1.54184 \AA$ ) radiation from a PhotonJet microfocus X-ray Source. Fourier Transformation Infrared spectra (FTIR) were recorded using a PerkinElmer Spectrum Two IR Spectrometer. UV-vis diffuse reflectance spectra were measured on an Ocean Insight USB2000+ miniature spectrometer with $\mathrm{BaSO}_{4}$ as the reflectance standard. Raman spectra were obtained on an QEPro high-performance Raman spectrometer (Ocean Insight) with $532 \mathrm{~nm}$ excitation. Freeze drying was performed using a CREATRUST CTFD-10P lyophiliser. Elemental analyses ( $\mathrm{C}$ and $\mathrm{H})$ were measured on a FLASH EA1112 elemental analyzer. The content of Ti(IV) of the TOCs was determined using the colorimetric method based on the characteristic UV-vis absorption of the yellow

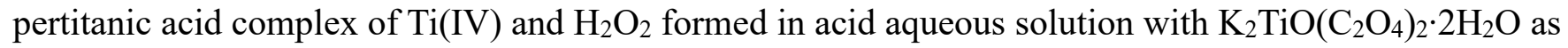
an external standard. The details were reported previously. ${ }^{1}$

Crystallography. A piece of crystal was mounted with an optical microscope under cryo- $\mathrm{N}_{2}$ steam. Data were collected at $173 \mathrm{~K}$ and processed using the CrystAlisPro software suite. ${ }^{2}$ The structures were solved and refined using the ShelXS and the ShelXL programs through the Olex2 1.3 software. ${ }^{3}$ Appropriate restraints were applied to the geometry. Hydrogen atoms at the carbon or nitrogen atoms were placed in the calculated positions and refined isotropically with the riding model. Solvent mask was conducted using the Olex2 implementation of BYPASS. The crystallographic data have been deposited as CCDC 20903762090382.

Synthesis of the compounds. Caution: Dissolution of $\mathrm{TiCl}_{4}$ in water emits lots of heat and corrosive acidic moisture and thus must be performed in a well-ventilated fume hood. One should wear gloves, 
safety goggles and face shields. Water should be added slowly and in portions. An ice-cold water bath should be used to cool the solution.

\section{$3,6,10,13,17,20,24,27$-octaaza-1,15(1,4)-dibenzenacyclooctacosaphane chloride (denoted as $\mathrm{C}_{30} \mathrm{~N} 8$ ).}

The protocol was modified from that reported by Winpenny et al. ${ }^{4}$ To a solution of N,N'-bis(2-aminoethyl)-1,3-propanediamine $(6.25 \mathrm{mmol})$ in $50 \mathrm{~mL}$ of methanol, 1,4-phthaladehyde $(6.20 \mathrm{mmol})$ in 30 $\mathrm{mL}$ of methanol was added very slowly and the reaction mixture was refluxed for $5 \mathrm{~h}$. Then, solid $\mathrm{NaBH}_{4}$ (5 equivalents) was added and the reaction mixture was stirred for $12 \mathrm{~h}$. The reaction was quenched with water and the solvent was removed by rotary evaporation. The resultant solid was extracted with chloroform, washed with water and dried over anhydrous magnesium sulphate, concentrated to $1 / 2$, and acidified with $\mathrm{HCl}$ (concentrated, $5 \mathrm{~mL}$ ). The resultant precipitate (obtained as chloride salt) was collected by vacuum filtration and wash with dichloromethane and diethyl ether to give after drying in vacuo at $50{ }^{\circ} \mathrm{C}$ a white power (yield $\approx 30 \%$ ). Further purification of the product was not performed.

N1-benzyl-N3-(3-(benzylamino)propyl)propane-1,3-diamine chloride $\left(\mathrm{C}_{8} \mathrm{~N}_{3} \mathrm{Ph}_{2}\right)$. Preparation as for $\mathbf{C}_{30} \mathbf{N}_{8}$, but replacing " $\mathrm{N}, \mathrm{N}$ '-bis(2-aminoethyl)-1,3-propanediamine (6.25 mmol) in $50 \mathrm{~mL}$ of methanol, 4phthalaldehyde $(0.66 \mathrm{~g}, 6.20 \mathrm{mmol})$ in $30 \mathrm{~mL}$ methanol" with "bis(3-aminopropyl)amine $(6.25 \mathrm{mmol})$ in $50 \mathrm{~mL}$ of methanol, benzaldehyde (13 $\mathrm{mmol})$ in $30 \mathrm{~mL}$ methanol".

\section{N1,N1'-(ethane-1,2-diyl)bis(N2-(pyridin-4-ylmethyl)ethane-1,2-diamine) chloride (denoted as}

$\mathbf{C}_{\mathbf{8}} \mathbf{N}_{6} \mathbf{P y}_{\mathbf{2}}$ ). Preparation as for $\mathbf{C}_{\mathbf{3 0}} \mathbf{N}_{\mathbf{8}}$, but replacing "N,N'-bis(2-aminoethyl)-1,3-propanediamine (6.25 $\mathrm{mmol})$ in $50 \mathrm{~mL}$ of methanol, 4-phthalaldehyde $(0.66 \mathrm{~g}, 6.20 \mathrm{mmol})$ in $30 \mathrm{~mL}$ methanol" with "TEtTA $(6.25 \mathrm{mmol})$ in $50 \mathrm{~mL}$ of methanol, pyridine-4-carboxaldehyde $(13 \mathrm{mmol})$ in $30 \mathrm{~mL}$ methanol”.

step 2, $\mathrm{NaBH}_{4}$




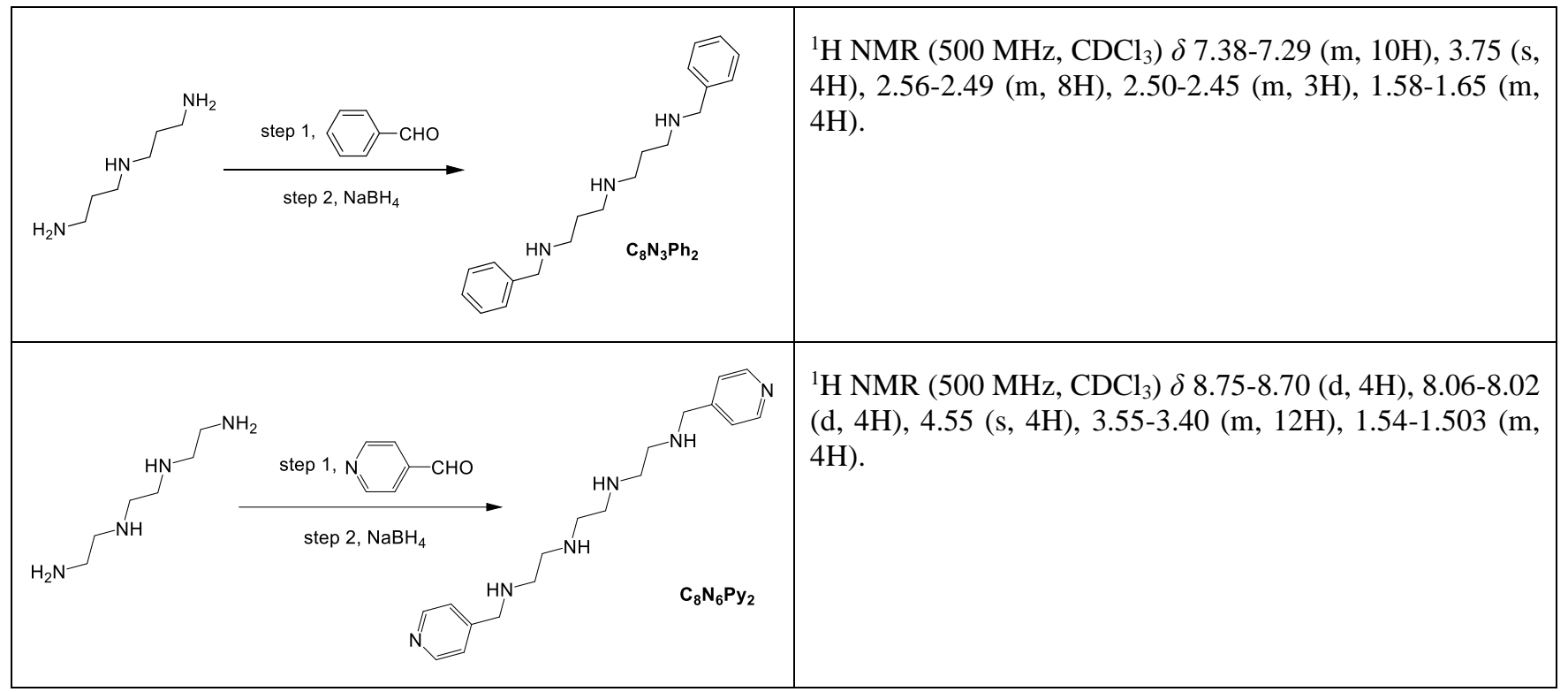

Synthesis of [Tis EtDA]. For the synthesis of the TOCs, only the optimized protocols were reported herein. A $10 \mathrm{~mL}$ Pyrex vial was charged with $2.0 \mathrm{~mL}$ of $1.0 \mathrm{M} \mathrm{TiCl}_{4}, 0.50 \mathrm{~mL}$ of $\mathrm{H}_{2} \mathrm{SO}_{4}$ (aq) and $0.50 \mathrm{mmol}$ of EtDA. Subsequently, the mixture was filtered with $0.22 \mu \mathrm{m}$ membrane followed by solvent evaporation at room temperature under ambient conditions. Colorless block crystals which formed after 3 days were separated using a Büchner funnel under suction, during which $40 \mathrm{~mL}$ of acetonitrile was used to wash the crystals. Finally, the crystals were stored in a sealed vial under ambient conditions before any purposes (yield $\approx 65 \%$ ). Anal. Calcd (\%): Ti, 13.3; C, 6.28; H, 3.87. Found (\%): Ti, 13.8; C, 6.20; H, 3.92.

Synthesis of $\left[\mathbf{T i}_{8} \cdot \mathrm{BuDA}\right]$. Preparation as for [Tis $\cdot$ EtDA], while both PrDA $(1.0 \mathrm{mmol})$ and BuDA $(0.50$ mmol) were used instead of EtDA. Colorless block crystals formed after ca. 5 days. However, only BuDA was found in compound [Tis.BuDA] (yield $\approx 68 \%$ ). Anal. Calcd (\%): Ti, 12.9; C, 8.08; H, 4.31. Found (\%): Ti, 13.0; C, 8.50; H, 3.75 .

Synthesis of [Ti8·TEtTA]. Preparation as for [Tis:EtDA], while replacing EtDA with 0.25 mmol of TEtTA. Colorless block crystals formed after ca. 7 days (yield $\approx 60 \%$ ). Anal. Calcd (\%): Ti, 13.7; C, 7.75; H, 3.66. Found (\%): Ti, 13.2; C, 7.95; H, 3.89 .

Synthesis of $[2 \mathbf{T i} \cdot$ spermine]. Preparation as for [Tis:EtDA], while replacing EtDA with $0.25 \mathrm{mmol}$ of spermine. Colorless block crystals formed after ca. 1 week (yield $\approx 60 \%$ ). Anal. Calcd (\%): Ti, 13.0; C, 11.3; H, 4.47. Found (\%): Ti, 12.9; C, 12.2; H, 4.21.

Synthesis of $\left[\mathbf{T i}_{8} \cdot \mathbf{C}_{8} \mathbf{N}_{6} \mathbf{P y}_{2}\right]$. Preparation as for [Ti8 $\cdot$ EtDA], while replacing " $2.0 \mathrm{~mL}$ of $1.0 \mathrm{M} \mathrm{TiCl}_{4}, 0.50$ 
$\mathrm{mL}$ of $\mathrm{H}_{2} \mathrm{SO}_{4}$ (aq) and $0.50 \mathrm{mmol}$ of EtDA." with "1.0 mL of $1.0 \mathrm{M} \mathrm{TiCl}_{4}, 0.25 \mathrm{~mL}$ of $\mathrm{H}_{2} \mathrm{SO}_{4}(\mathrm{aq})$ and 0.20 mmol of $\mathbf{C}_{\mathbf{8}} \mathbf{N}_{6} \mathbf{P y}$." Colorless block crystals which formed after ca. 2 weeks (yield $\approx 35 \%$ ). Anal. Calcd (\%): Ti, 12.4; C, 14.0; H, 3.57. Found (\%): Ti, 11.6; C, 13.9; H, 3.95.

Synthesis of $\left[\mathbf{T i}_{8} \cdot \mathbf{C}_{8} \mathbf{N}_{6} \mathbf{P h}_{2}\right]$. Preparation as for [Tis. EtDA], while replacing "2.0 $\mathrm{mL}$ of $1.0 \mathrm{M} \mathrm{TiCl}_{4}, 0.50$ $\mathrm{mL}$ of $\mathrm{H}_{2} \mathrm{SO}_{4}(\mathrm{aq})$ and $0.50 \mathrm{mmol}$ of EtDA." with "1.0 mL of $1.0 \mathrm{M} \mathrm{TiCl}_{4}, 0.25 \mathrm{~mL}$ of $\mathrm{H}_{2} \mathrm{SO}_{4}$ (aq) and 0.10 mmol of $\mathbf{C}_{8} \mathbf{N}_{6} \mathbf{P h}_{2}$." Colorless block crystals formed after 20 days (yield $\approx 30 \%$ ). Anal. Calcd (\%): Ti, 8.40; C, 26.4; H, 4.96. Found (\%): Ti, 8.60; C, 25.8; H, 4.86.

Synthesis of $\left[\mathbf{T i}_{8} \cdot \mathbf{C}_{30} \mathbf{N} 8\right.$ ]. Preparation as for [Tis.EtDA], while replacing while replacing " $2.0 \mathrm{~mL}$ of 1.0 $\mathrm{M} \mathrm{TiCl}_{4}, 0.50 \mathrm{~mL}$ of $\mathrm{H}_{2} \mathrm{SO}_{4}(\mathrm{aq})$ and $0.50 \mathrm{mmol}$ of EtDA." with " $1.0 \mathrm{~mL}$ of $1.0 \mathrm{M} \mathrm{TiCl}_{4}, 0.25 \mathrm{~mL}$ of $\mathrm{H}_{2} \mathrm{SO}_{4}(\mathrm{aq})$ and $0.10 \mathrm{mmol}$ of $\mathbf{C}_{\mathbf{3 0}} \mathbf{N 8}$ ". Colorless block crystals formed after ca. 1 month (yield $\approx 20 \%$ ). Anal. Calcd (\%): Ti, 12.4; C, 11.6; H, 3.64. Found (\%): Ti, 12.7; C, 12.3; H, 3.25. 
The table of crystallographic data

\begin{tabular}{|c|c|c|c|}
\hline Compound & {$\left[\mathbf{T i}_{8} \cdot \mathrm{EtDA}\right]$} & {$\left[\mathbf{T i}_{8} \cdot \mathrm{BuDA}\right]$} & {$\left[\mathbf{T i}_{\mathbf{8}} \cdot \mathrm{TEtTA}\right]$} \\
\hline Formula unit & $\mathrm{Ti}_{8} \mathrm{O}_{91} \mathrm{~S}_{16.5} \mathrm{C}_{15} \mathrm{~N}_{15} \mathrm{H}_{111}$ & $\mathrm{Ti}_{8} \mathrm{O}_{98} \mathrm{~S}_{16} \mathrm{C}_{20} \mathrm{~N}_{10} \mathrm{H}_{128}$ & $\mathrm{Ti}_{8} \mathrm{O}_{88} \mathrm{~S}_{16} \mathrm{C}_{18} \mathrm{~N}_{12} \mathrm{H}_{102}$ \\
\hline CCDC number & 2090376 & 2090377 & 2090378 \\
\hline Moieties & {$\left[\mathrm{Ti}_{8} \mathrm{O}_{8}\left(\mathrm{SO}_{4}\right)_{14}\left(\mathrm{HSO}_{4}\right)_{2}\right]\left(\mathrm{C}_{2} \mathrm{H}_{10} \mathrm{~N}_{2}\right)_{7.5}\left(\mathrm{SO}_{4}\right)_{0.5} \cdot 17 \mathrm{H}_{2} \mathrm{O}$} & {$\left[\mathrm{Ti}_{8} \mathrm{O}_{8}\left(\mathrm{SO}_{4}\right)_{10}\left(\mathrm{HSO}_{4}\right)_{6}\right]\left(\mathrm{C}_{4} \mathrm{H}_{14} \mathrm{~N}_{2}\right)_{5} \cdot 26 \mathrm{H}_{2} \mathrm{O}$} & {$\left[\mathrm{Ti}_{8} \mathrm{O}_{8}\left(\mathrm{SO}_{4}\right)_{12}\left(\mathrm{HSO}_{4}\right)_{4}\right]\left(\mathrm{C}_{6} \mathrm{H}_{20} \mathrm{~N}_{4}\right)_{3} \cdot 16 \mathrm{H}_{2} \mathrm{O}$} \\
\hline Formula weight (g/mol) & 2867.4 & 2970.4 & 2788.4 \\
\hline Crystal system & monoclinic & orthorhombic & monoclinic \\
\hline Space group (Nr.) & $P 2_{1} / n$ & Pnma & $P 2_{1} / n$ \\
\hline $\mathrm{a}(\AA)$ & $17.1375(3)$ & $18.5317(3)$ & $19.9453(2)$ \\
\hline $\mathrm{b}(\AA)$ & $37.0542(5)$ & $28.5245(4)$ & $15.9411(2)$ \\
\hline c $(\AA)$ & $17.3720(3)$ & $19.8526(3)$ & $30.1272(3)$ \\
\hline$\alpha\left(^{\circ}\right)$ & 90 & 90 & 90 \\
\hline$\beta\left(^{\circ}\right)$ & $114.321(2)$ & 90 & $96.2410(10)$ \\
\hline$\gamma\left({ }^{\circ}\right)$ & 90 & 90 & 90 \\
\hline Volume $\left(\AA^{3}\right)$ & $10052.5(3)$ & $10494.2(3)$ & $9522.17(18)$ \\
\hline $\mathrm{Z}$ & 4 & 4 & 4 \\
\hline Density $_{\text {calc }}\left(\mathrm{g} / \mathrm{cm}^{3}\right)$ & 1.794 & 1.582 & 1.810 \\
\hline Abs. Coeff. $\mu\left(\mathrm{mm}^{-1}\right)$ & 10.048 & 8.850 & 9.837 \\
\hline Temperature (K) & $172.99(10)$ & $172.99(10)$ & $172.99(10)$ \\
\hline Total reflections & 64709 & 33500 & 55646 \\
\hline Min-max $2 \theta\left(^{\circ}\right)$ & 2.794 to 53.186 & 5.424 to 153.406 & 6.282 to 153.062 \\
\hline Unique reflections & 19728 & 10558 & 18788 \\
\hline $\mathrm{R}_{1}[\mathrm{I}>=2 \sigma]$ & 0.0688 & 0.1161 & 0.0805 \\
\hline$w_{2}$ (all data) & 0.2046 & 0.3194 & 0.2400 \\
\hline $\mathrm{R}_{\text {int }}$ & 0.0528 & 0.0884 & 0.0577 \\
\hline Goodness of fit on $\mathrm{F}^{2}$ & 1.044 & 1.092 & 1.087 \\
\hline Parameters & 1289 & 643 & 1468 \\
\hline Restraints & 184 & 270 & 758 \\
\hline Largest diff. peak/hole $\left(\mathrm{e} \AA^{-3}\right)$ & $1.97 /-1.85$ & $1.45 /-1.55$ & $2.23 /-1.48$ \\
\hline
\end{tabular}




\begin{tabular}{|c|c|c|}
\hline Compound & [2Tis:spermine] & {$\left[\mathrm{Ti}_{8} \cdot \mathrm{C}_{8} \mathrm{~N}_{6} \mathrm{Py}_{2}\right]$} \\
\hline Formula unit & $\mathrm{Ti}_{8} \mathrm{O}_{96} \mathrm{~S}_{16} \mathrm{C}_{30} \mathrm{~N}_{12} \mathrm{H}_{142.67} \mathrm{Cl}_{0.67}$ & $\mathrm{Ti}_{8} \mathrm{O}_{88} \mathrm{~S}_{16} \mathrm{C}_{36} \mathrm{~N}_{12} \mathrm{H}_{110} \mathrm{Cl}_{2}$ \\
\hline CCDC number & 2090379 & 2090380 \\
\hline Moieties & {$\left[\mathrm{Ti}_{8} \mathrm{O}_{8}\left(\mathrm{SO}_{4}\right)_{12}\left(\mathrm{HSO}_{4}\right)_{4}\right]\left(\mathrm{C}_{10} \mathrm{H}_{30} \mathrm{~N}_{4}\right)_{3} \cdot 24 \mathrm{H}_{2} \mathrm{O} \cdot 0.67 \mathrm{HCl}$} & {$\left[\mathrm{Ti}_{8} \mathrm{O}_{8}\left(\mathrm{SO}_{4}\right)_{8}\left(\mathrm{HSO}_{4}\right)_{8}\right]\left(\mathrm{C}_{18} \mathrm{H}_{34} \mathrm{~N}_{6}\right)_{2} \cdot 16 \mathrm{H}_{2} \mathrm{O} \cdot 2 \mathrm{HCl}$} \\
\hline Formula weight $(\mathrm{g} / \mathrm{mol})$ & 3188.9 & 3083.4 \\
\hline Crystal system & triclinic & triclinic \\
\hline Space group (Nr.) & $P-1$ & $P-1$ \\
\hline $\mathrm{a}(\AA)$ & $16.4955(2)$ & $16.5361(4)$ \\
\hline$b(\AA)$ & $32.4208(3)$ & $16.8328(4)$ \\
\hline$c(\AA)$ & $34.4512(4)$ & $22.6839(5)$ \\
\hline$\alpha\left({ }^{\circ}\right)$ & $107.9050(10)$ & $92.2774(17)$ \\
\hline$\beta\left(^{\circ}\right)$ & $91.9770(10)$ & $104.8398(18)$ \\
\hline$\gamma\left({ }^{\circ}\right)$ & $100.2390(10)$ & $118.317(2)$ \\
\hline Volume $\left(\AA^{3}\right)$ & $17174.5(3)$ & $5275.7(2)$ \\
\hline $\mathrm{Z}$ & 6 & 2 \\
\hline Density $_{\text {calc }}\left(\mathrm{g} / \mathrm{cm}^{3}\right)$ & 1.567 & 1.755 \\
\hline Abs. Coeff. $\mu\left(\mathrm{mm}^{-1}\right)$ & 8.308 & 9.343 \\
\hline Temperature (K) & $173.00(10)$ & $172.99(10)$ \\
\hline Total reflections & 176240 & 52636 \\
\hline $\operatorname{Min}-\max 2 \theta\left(^{\circ}\right)$ & 5.47 to 153.386 & 2.796 to 53.196 \\
\hline Unique reflections & 67901 & 20730 \\
\hline $\mathrm{R}_{1}[\mathrm{I}>=2 \sigma]$ & 0.0996 & 0.0752 \\
\hline $\mathrm{wR}_{2}$ (all data) & 0.3141 & 0.2346 \\
\hline $\mathrm{R}_{\text {int }}$ & 0.0755 & 0.0649 \\
\hline Goodness of fit on $\mathrm{F}^{2}$ & 1.076 & 1.086 \\
\hline Parameters & 4002 & 1419 \\
\hline Restraints & 1244 & 332 \\
\hline Largest diff. peak/hole $\left(\mathrm{e} \AA^{-3}\right)$ & $5.02 /-1.39$ & $1.65 /-1.12$ \\
\hline
\end{tabular}




\begin{tabular}{|c|c|c|}
\hline Compound & {$\left[\mathrm{Ti}_{8} \cdot \mathrm{C}_{8} \mathrm{~N}_{3} \mathrm{Ph}_{2}\right]$} & {$\left[\mathrm{Ti}_{8} \cdot \mathrm{C}_{30} \mathrm{~N}_{8}\right]$} \\
\hline Formula unit & $\mathrm{Ti}_{8} \mathrm{O}_{120} \mathrm{~S}_{18} \mathrm{C}_{100} \mathrm{~N}_{15} \mathrm{H}_{226} \mathrm{Cl}$ & $\mathrm{Ti}_{8} \mathrm{O}_{98} \mathrm{~S}_{17} \mathrm{C}_{30} \mathrm{~N}_{8} \mathrm{H}_{112.5} \mathrm{Cl}_{0.5}$ \\
\hline CCDC number & 2090381 & 2090382 \\
\hline Moieties & $\begin{array}{l}{\left[\mathrm{Ti}_{8} \mathrm{O}_{8}\left(\mathrm{SO}_{4}\right)_{11}\left(\mathrm{HSO}_{4}\right)_{5}\right]\left(\mathrm{C}_{20} \mathrm{H}_{32} \mathrm{~N}_{3}\right)_{5}\left(\mathrm{SO}_{4}\right)_{2} \cdot 30 \mathrm{H}_{2} \mathrm{O}} \\
\mathrm{HCl}\end{array}$ & {$\left[\mathrm{Ti}_{8} \mathrm{O}_{8}\left(\mathrm{SO}_{4}\right)_{8}\left(\mathrm{HSO}_{4}\right)_{8}\right]\left(\mathrm{C}_{30} \mathrm{H}_{60} \mathrm{~N}_{8}\right)\left(\mathrm{SO}_{4}\right) \cdot 22 \mathrm{H}_{2} \mathrm{O} \cdot 0.5(\mathrm{HCl})$} \\
\hline Formula weight $(\mathrm{g} / \mathrm{mol})$ & 4549.9 & 3097.7 \\
\hline Crystal system & triclinic & triclinic \\
\hline Space group (Nr.) & $P-1$ & $P-1$ \\
\hline $\mathrm{a}(\AA)$ & 20.8813(7) & $16.9361(4)$ \\
\hline $\mathrm{b}(\AA)$ & $21.7966(7)$ & $17.2962(4)$ \\
\hline$c(\AA)$ & $22.1668(8)$ & $20.1240(5)$ \\
\hline$\alpha\left(^{\circ}\right)$ & $79.147(3)$ & $92.6637(19)$ \\
\hline$\beta\left(^{\circ}\right)$ & $79.248(3)$ & $106.676(2)$ \\
\hline$\gamma\left({ }^{\circ}\right)$ & $65.356(3)$ & $96.1545(19)$ \\
\hline Volume $\left(\AA^{3}\right)$ & $8939.8(6)$ & $5595.9(2)$ \\
\hline $\mathrm{Z}$ & 2 & 2 \\
\hline Density $_{\text {calc }}\left(\mathrm{g} / \mathrm{cm}^{3}\right)$ & 1.470 & 1.637 \\
\hline Abs. Coeff. $\mu\left(\mathrm{mm}^{-1}\right)$ & 5.840 & 8.694 \\
\hline Temperature $(\mathrm{K})$ & $172.98(10)$ & $172.99(10)$ \\
\hline Total reflections & 88147 & 56342 \\
\hline $\operatorname{Min}-\max 2 \theta\left(^{\circ}\right)$ & 5.052 to 153.73 & 2.374 to 53.232 \\
\hline Unique reflections & 34816 & 22029 \\
\hline $\mathrm{R}_{1}[\mathrm{I}>=2 \sigma]$ & 0.1648 & 0.1124 \\
\hline $\mathrm{wR}_{2}$ (all data) & 0.4872 & 0.3358 \\
\hline $\mathrm{R}_{\mathrm{int}}$ & 0.1860 & 0.0769 \\
\hline Goodness of fit on $\mathrm{F}^{2}$ & 1.201 & 1.078 \\
\hline Parameters & 2071 & 1409 \\
\hline Restraints & 875 & 612 \\
\hline Largest diff. peak/hole $\left(e \AA^{-3}\right)$ & $1.82 /-1.53$ & $3.98 /-1.89$ \\
\hline
\end{tabular}




\section{Supplementary Data and Discussion}

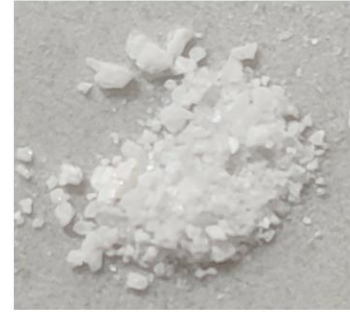

$\left[\mathrm{Ti}_{8} \cdot\right.$ EtDA]

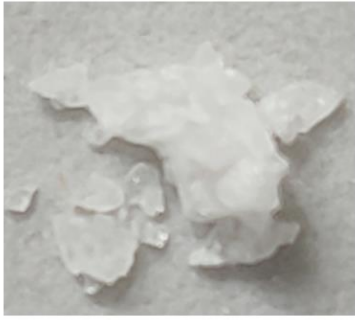

$\left[\mathrm{Ti}_{8} \cdot \mathrm{BuDA}\right]$

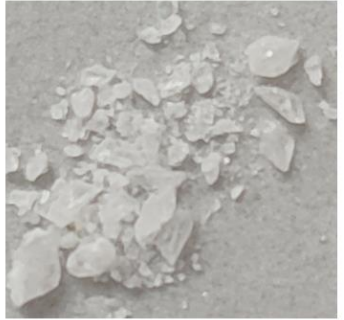

$\left[\mathrm{Ti}_{8} \cdot \mathrm{TEtTA}\right]$

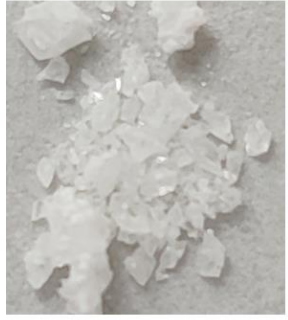

[2 $\mathrm{Ti}_{8} \cdot$ spermine]

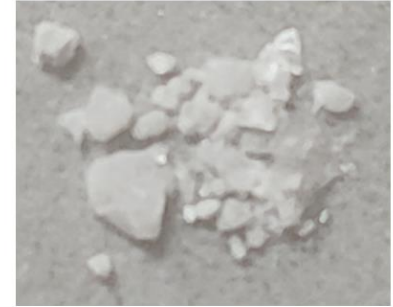

$\left[\mathrm{Ti}_{8} \cdot \mathrm{C}_{8} \mathrm{~N}_{4} \mathrm{Py}_{2}\right]$

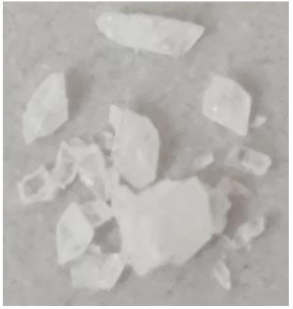

$\left[\mathrm{Ti}_{8} \cdot \mathrm{C}_{8} \mathrm{~N}_{3} \mathrm{Ph}_{2}\right]$

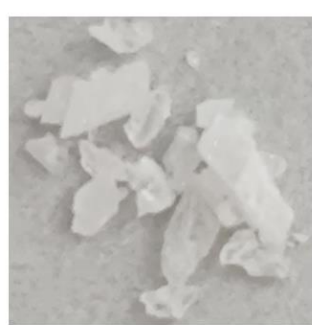

$\left[\mathrm{Ti}_{8} \cdot \mathrm{C}_{30} \mathrm{~N}_{8}\right]$

Figure S1. The photos of the compounds.

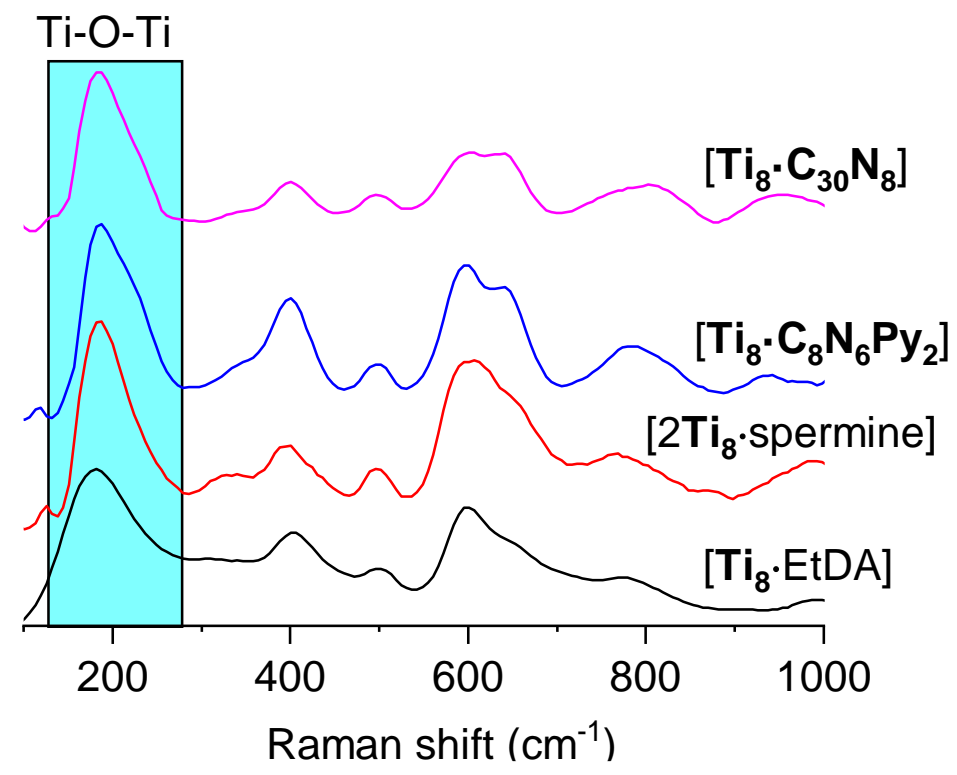

Figure S2. Raman spectra of the compounds. 


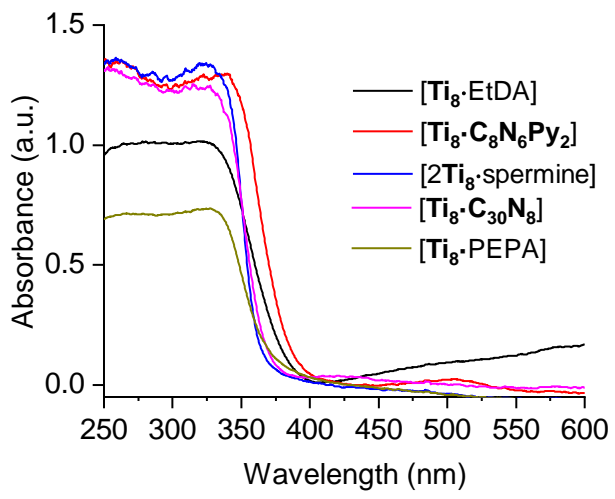

Figure S3. Diffuse reflectance UV-vis spectra of the compounds. The band absorption edge of these compounds ranges $390-400 \mathrm{~nm}$.
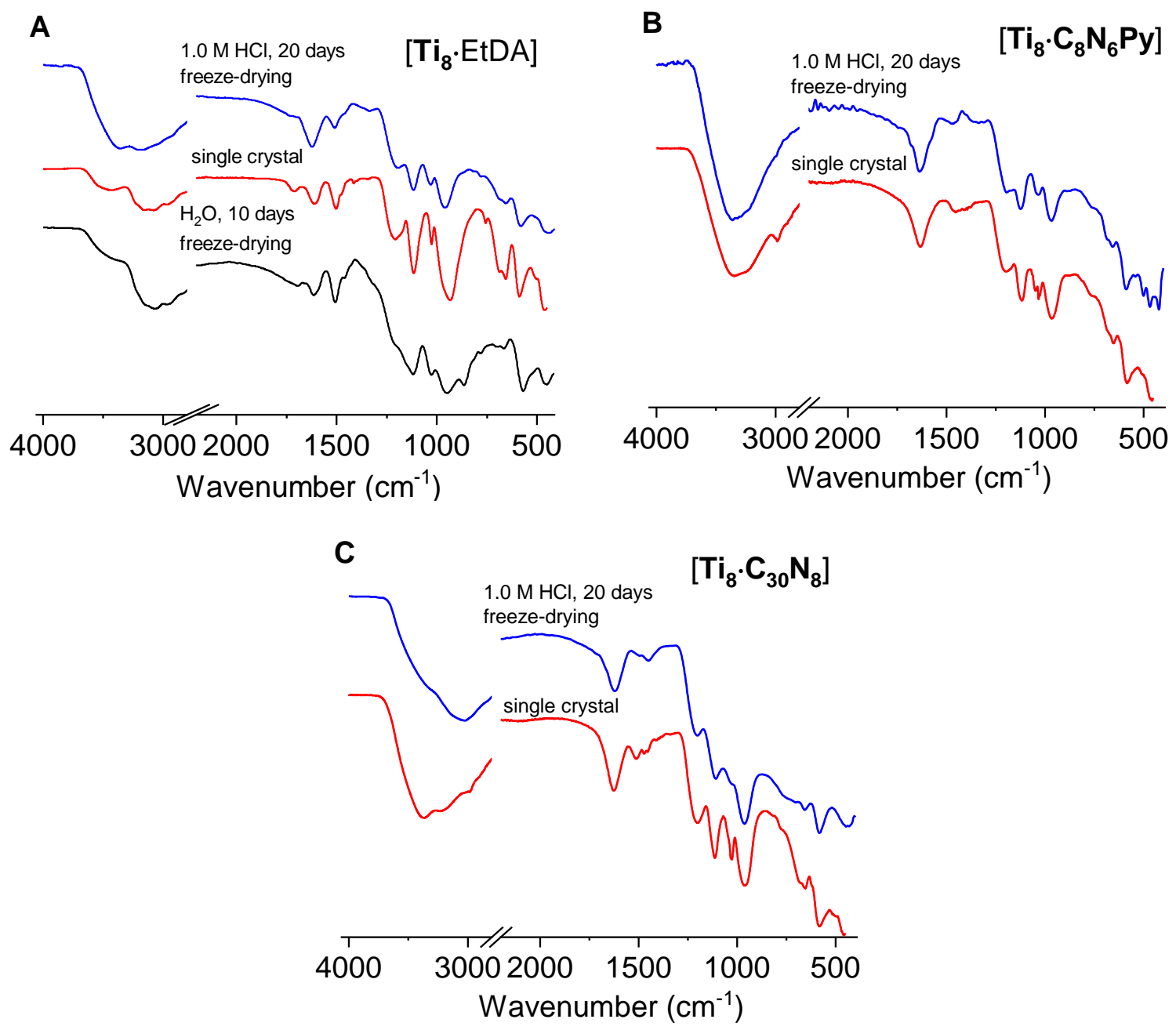

Figure S4. IR spectra of (A) [ $\left.\mathbf{T i}_{8} \cdot \mathrm{EtDA}\right]$, (B) $\left[\mathbf{T i}_{8} \cdot \mathbf{C}_{\mathbf{8}} \mathbf{N}_{6} \mathbf{P} \mathbf{y}_{2}\right]$ and (C) $\left[\mathbf{T i}_{8} \cdot \mathbf{C}_{30} \mathbf{N}_{8}\right]$ in $1.0 \mathrm{M} \mathrm{HCl}, 20$ days or in $\mathrm{H}_{2} \mathrm{O}$, 10 days after freeze-drying. The results show that they are stable in acidic aqueous solution.

Discussion. In panel A, the IR of [ $\mathbf{T i}_{8} \cdot$ EtDA] indicates most of it decomposed after 10 days in neutral water (some features of $\left[\mathbf{T i}_{\mathbf{8}} \cdot \mathrm{EtDA}\right]$ could be disernable). 

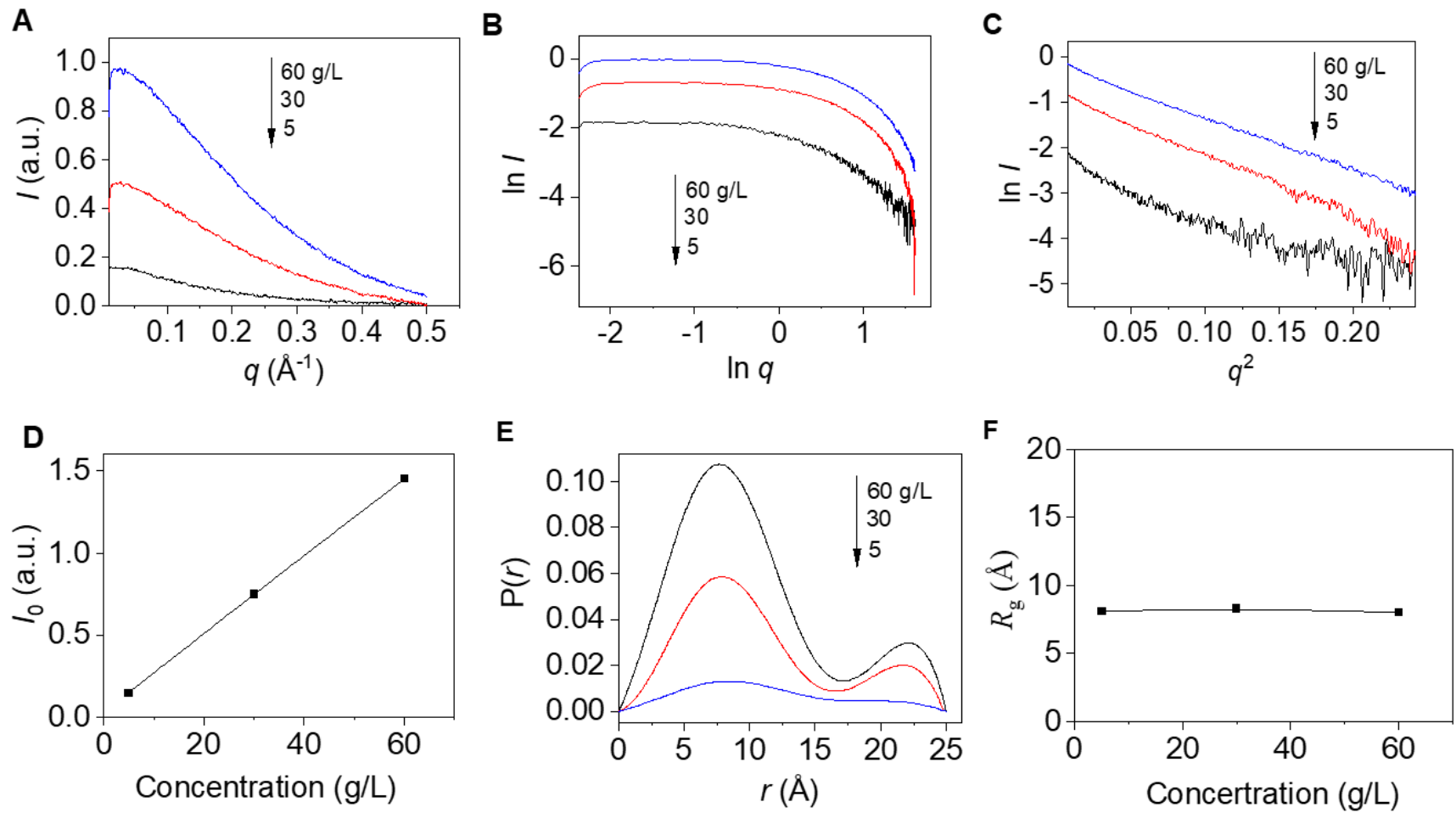

Figure S5. SAXS data for $\left[\mathbf{T i}_{\mathbf{8}} \cdot \mathrm{EtDA}\right]$ in water.

Discussion. Since acid could cause damage to the instrument, [ $\left.\mathbf{T i}_{\mathbf{8}} \cdot \mathrm{EtDA}\right]$ was dissolved in neutral water for SAXS measurements. SAXS data were analyzed following the literature method. ${ }^{5}$ Panel A shows the experimental data after subtraction of background $\left(\mathrm{H}_{2} \mathrm{O}\right)$. Figure $\mathrm{S} 5 \mathrm{~A}$ is transformed into Figure $\mathrm{S} 5 \mathrm{~B}$ to convey information about the size of scattering features. After that, the correlation between $\ln I$ and $\mathrm{q}^{2}$ (low-q region) is plotted as the Guinier curve in Figure S5C. Finally, the important parameters including extrapolated zero-angle intensity $\left(\mathrm{I}_{0}\right.$, panel $\left.\mathrm{D}\right)$, pair distance distribution function $(\mathrm{P}(\mathrm{r})$, panel $\mathrm{E})$, and radius of gyration ( $\mathrm{Rg}$, panel F) are calculated using the SAS Data Analysis package of ATSAS 2.6.0. As the scattering intensity $\mathrm{I}_{0}$ is proportional to concentration (panel D) and the radius of gyration $\mathrm{R}_{\mathrm{g}}$ does not vary with concentration (panels $\mathrm{E}$ and $\mathrm{F}$ ), the solution speciation must maintain as concentration varies. In panel $\mathrm{E}, \mathrm{P}(\mathrm{r})$ is the probability of finding the vector length $\mathrm{r}$ in the molecule that will become zero at the maximum vector length. Hence, the two peaks in panel E agree with a $17-\AA \mathbf{T i}_{8}$ ring (hydrodynamic diameter) surrounded with a shell of counterions. The diameter of $\mathbf{T i}_{\mathbf{8}}$ ring by the SAXS data agrees well with its crystallographic dimension (recall Figure 1). The thickness of the shell of the counterion (ca. $8 \AA$ ) is well consistent with a previous report by Pigga et al. in which the counterion distribution around the $\left\{\mathrm{Mo}_{72} \mathrm{~V}_{30}\right\}$ macroanion was determined using SAXS. ${ }^{6}$ In all, the SAXS data suggest the Ti8 ring of [Ti8$\cdot$ EtDA] is stable when dissolved in $\mathrm{H}_{2} \mathrm{O}$. 

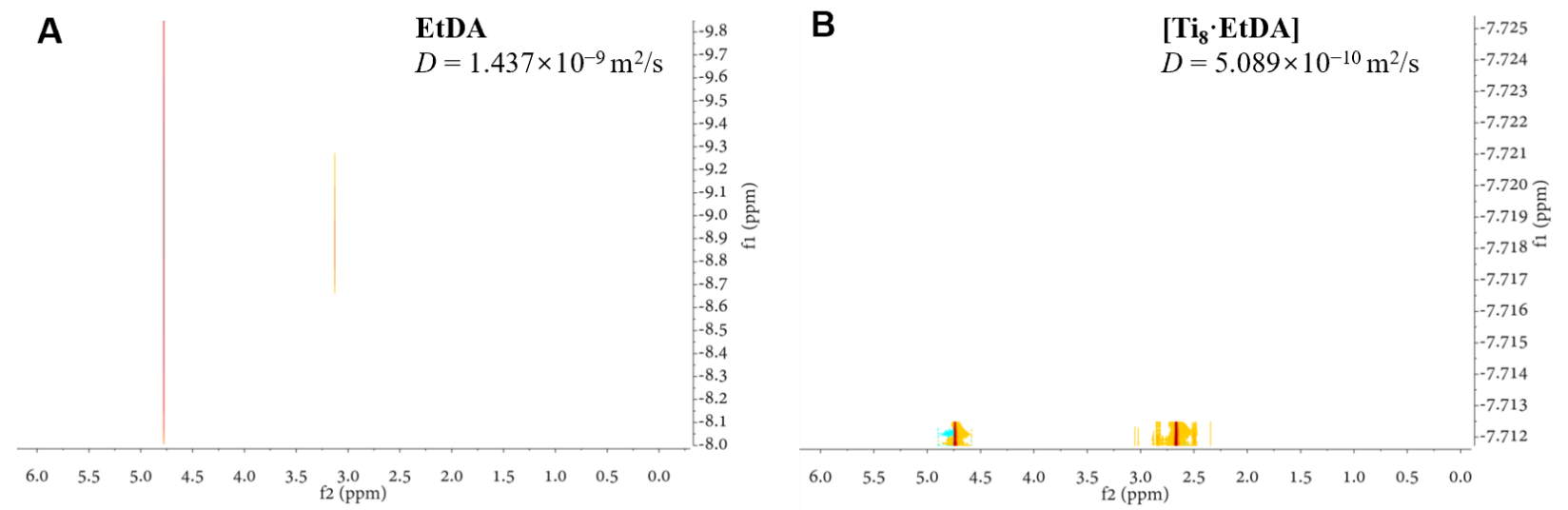

Figure S6. ${ }^{1} \mathrm{H}$ DOSY NMR of EtDA and [Ti; $\cdot$ EtDA] $(400 \mathrm{MHz})$. The solutions were prepared using $1.0 \mathrm{M} \mathrm{HCl}$ in $\mathrm{D}_{2} \mathrm{O}$.

Discussion. From the ${ }^{1} \mathrm{H}$ DOSY NMR spectra, the diffusion coefficient $(D)$ value of EtDA in $1.0 \mathrm{M} \mathrm{HCl}$ $\left(1.4 \times 10^{-9} \mathrm{~m}^{2} \mathrm{~s}^{-1}\right)$ is much larger than that in a solution of $\left[\mathbf{T i}_{\mathbf{8}} \cdot\right.$ EtDA] $\left(5.1 \times 10^{-9} \mathrm{~m}^{2} \mathrm{~s}^{-1}\right)$. The decreased mobility of EtDA is attributed to the confinement by the Ti.8 ring and suggests EtDA might be hosted by and associated with $\mathbf{T i}_{\mathbf{8}}$ in the solution. 


\section{References}

1. Zhang, G.; Hou, J.; Tung, C.-H.; Wang, Y., Small Titanium Oxo Clusters: Primary Structures of Titanium(IV) in Water. Inorg. Chem. 2016, 55 (7), 3212-3214.

2. Sheldrick, G. M. SADABS, A Program for Empirical Absorption Correction; University of Göttingen: Göttingen, Germany, 1998.

3. Dolomanov, O. V.; Bourhis, L. J.; Gildea, R. J.; Howard, J. A. K.; Puschmann, H., Olex2: a Complete Structure Solution, Refinement and Analysis Program. J. Appl. Crystallogr. 2009, 42 (2), 339-341.

4. Timco, G. A.; Fernandez, A.; Kostopoulos, A. K.; Charlton, J. F.; Lockyer, S. J.; Hailes, T. R.; Adams, R. W.; McInnes, E. J. L.; Tuna, F.; Vitorica-Yrezabal, I. J.; Whitehead, G. F. S.; Winpenny, R. E. P., Hybrid Organic-Inorganic Rotaxanes, Including a Hetero-Hybrid [3]Rotaxane Featuring Two Distinct Heterometallic Rings and a Molecular Shuttle. Angew. Chem. Int. Ed. 2018, 57 (34), 10919-10922.

5. (a) Fullmer, L. B.; Molina, P. I.; Antonio, M. R.; Nyman, M., Contrasting Ion-association Behaviour of Ta and Nb Polyoxometalates. Dalton. Trans. 2014, 43 (41), 15295-9; (b) Antonio, M. R.; Nyman, M.; Anderson, T. M., Direct Observation of Contact Ion-Pair Formation in Aqueous Solution. Angew. Chem. Int. Ed. 2009, 48 (33), 6136-6140; (c) Hou, Y.; Zakharov, L. N.; Nyman, M., Observing Assembly of Complex Inorganic Materials from Polyoxometalate Building Blocks. J. Am. Chem. Soc. 2013, 135 (44), 16651-16657; (d) O. Sadeghi, L. N. Z., M. Nyman, Aqueous Formation and Manipulation of the Iron-oxo Keggin Ion. Science 2015, 347, 1359-1362.

6. $\quad$ Pigga, J. M.; Kistler, M. L.; Shew, C. Y.; Antonio, M. R.; Liu, T., Counterion Distribution Around Hydrophilic Molecular Macroanions: the Source of the Attractive Force in Self-assembly. Angew. Chem. Int. Ed. Engl. 2009, 48 (35), 6538-42. 